\title{
Kirsten Drotner \& Kim Christian Schrøder (eds.): Digital Content Creation - Perceptions, Practices \& Perspectives. New literacies and digital epistemologies Series, Vol. 46. New York: Peter Lang. 2010.
}

\author{
Martin Brynskov
}

MedieKultur 2011, 50, 197-201

Published by SMID | Society of Media researchers In Denmark | www.smid.dk The online version of this text can be found open access at www.mediekultur.dk

The fast-paced evolution of digital technologies and their formative role in knowledgebased societies represent a daunting challenge to describe, let alone to understand in more depth. Digital Content Creation is an anthology which, in its own words, offers "sobering perspectives" based on theory-driven empirical research. It aims to take stock of broad developments within social media through reasoned and robust studies, primarily of innovative uses of young people's creative forms of content creation.

To this end, a variety of traditions - including media and ICT studies, education, psychology, anthropology, sociology and cultural studies - form an analytical perspective based on situated praxis and empirical complexity, in explicit opposition to more descriptive and controlled approaches. This can hardly be a surprise, given the name and history of the book series, New Literacies and Digital Epistemologies, but it is very welcome to see an attempt to not only frame the discussion of social media, but also to offer some answers that combine the detailed lens of empirical studies with broader conceptual categories such as policy-making, commercial issues and the position of scholarly contributions.

Especially refreshing is the explicit discussion of the meta-issue of the role of scholarship in an area that finds itself in such a compressed space between rapid technological development and business innovation on one side, and wide distribution and profound societal impact on the other. How is it possible to say anything well-founded that isn't already outdated by the time it is published? The response to this challenge is tied directly to the type of interventions and contributions the authors offer: strongly focused analytical means to 
dig nuggets of insight out of empirical complexities. The results may not be immediately ready for broader generalization, but their purpose is to try to develop the field, a field with "no easy answers or simple outcomes".

Let us look at some of these investigations. The book is divided into five parts and consists of 15 chapters, all but one originating in the 2008 DREAM conference on Digital Content Creation.

Part one, "Digital Content Creation: Creative Processes and Textual Re-appropriations", revolves around the relation between remix culture and design processes. The perspectives are treated both as more abstract phenomena, with cultural and professional histories, and as elements of mundane praxis in education. The underlying argument is that remixing is a valid perspective that needs to be developed and leveraged more systematically in education. However, viewing education as a design process requires a great deal of experimentation and often challenges existing boundaries established through the history of formal education. The fabric, as it were, of social media offers new challenges to basic concepts such as ownership and authorship, the purpose of education as it is practiced, and the freedom of media choice.

The authors in this section argue strongly that the process of learning in itself is foregrounded, most markedly by Löwgren's assertion (in “Designing for Collaborative Crossmedia Creation") that to successfully facilitate cross-media creation, the designer/educator is wise not to focus on outcomes at all, only on shaping the processes in certain specific ways. That is how s/he will leverage the qualities of social media in education, because many of the qualities are emergent features rather than carefully designable discrete elements.

The studies presented in this section range from historical perspectives (Erstad: "Content in Motion") and small-scale interventions (Clark: "Wiki as Semiospheric Text") to a large corporate development project (Staffans \& Wiklund-Engblom: "Developing Crossmedia and Interactivity for Edutainment).

Part two, "Competence Formation through Digital Content Creation: Personal Voice or Commercial Coercion?", addresses the tension between critical and commercial values in platforms of creative expression. Here the chapters converge on a pragmatic stance on the issue, from Hartley's "Where Money and Meanings Meet", which argues for a new paradigm for digital literacy, essentially a reconciliation of the economic and cultural spheres under the term cultural science, to Stephenson's "Media Production and the Problem of Consumption", highlighting insights gained from failure to combine adult-driven discourses and youth practices in critical media production in the classroom. Haferkamp and Krämer ("Creating a Digital Self") describe a qualitative study on how children use social networking sites for identity construction, including paradoxes in what they do themselves and which motives they ascribe to others.

Hartley unfolds an analytical framework of cultural science to understand and address the characteristics of digital media, and thus his contribution is theoretical in nature, linking digital media to the Westernised chain of media evolution: print, media, critical and digital. 
Stephenson, on the other hand, delivers three recommendations to educators, emerging from failed practice, the last of which is that "messing around" or tinkering with digital media production tools is crucial and should be given specific time when planning courses. On a side note, this resonates with other experiences from efforts to combine digital technologies and design thinking at university level education.

Part three, "Institutional Ramifications of Digital Content Creation for Learning", follows up on the previous section with three chapters that look at digital creativity and learning from an institutional perspective. In "Digital Creativity", McFarlane asserts what Stephenson experienced: there is still a lack of practice-based knowledge on how to integrate digital learning as part of formal, general education. Trying to explain why digital learning is still struggling, she points out that teaching digital literacy - with its iterative, remixed nature lends itself to a constructivist approach. But contrary to much STEM (science/technology/ engineering/mathematics) pedagogy, McFarlane warns about overreliance on Vygotsky's scaffolding principle, and characterises Prensky's notion of the "digital native" as being risky; the former because it downplays the role of peer interaction, the latter because it ignores the seemingly majority of children who do not develop digital literacy without further instruction. While not explicitly taking a design perspective, she proposes learning methods based on iterative, productive tasks that include reflection, critique and development in an active community of co-learners. This does indeed smack of "design thinking", pointing to an underlying current in many of the concrete recommendations throughout the book.

Grønning ("Chat Interactions in Public and Commercial Domains") takes a non-educational perspective on the perception of chat (instant messaging, IM) within two organisational contexts: a public education guide service and IKEA customer service. The findings include a general characterisation of chat as an emerging medium in professional relations, and points to concrete patterns of chat offering lower barriers of entry compared to email or physical interaction for young males. In "Learning Space(s)", Weight offers seven concrete questions for course designers with regard to digital contexts for learning derived from a case study of university teaching. A handy, relevant and easy read for anyone in that situation, it is also based on a constructivist view.

Part four, "Studying Digital Content Creation", turns to look at methodological challenges in studies of creative digital media use in teaching. The first chapter, by Barthel, Ainsworth and Sharples ("Negotiating Perspectives in Social Video Environments"), is a case study of the design process of a video editing tool and its use as an educational resource for knowledge building with video. The chapter is a classical design case, but the focus on the method aims to draw some more general conclusions based on a formative evaluation and knowledge building theory. The conclusions are there, including a call for theory-based design of educational software; however, as a reader, it is difficult to assess these claims since we are told very little about the actual results from the analyses.

The next chapter, Spikols' “Designing Strategies for Developing Mobile Collaborative Learning Platforms", is another design-oriented case study, a mobile game for learning 
activities. The author goes a long way to set the context for both design-based research and mobile learning in general, and to document the actual design process. It is a very good example of the challenges facing design-based research in terms of methodology, but at the same time, it shows the value of design-based research - a notion that is echoed throughout the book. Exactly these kinds of studies are needed to get a clearer understanding of the design and use of digital learning platforms, mobile being an increasingly important one. In “Digitally Mapping a Rhizome”, Kivelä and Mylläri report on an attempt to map physical and digital footprints in a science class setting. They discuss how to represent the heterogeneous representations captured during the digital content creation and what insights may be obtained based on such mappings. Their conclusions are mainly about how to get the best data (e.g., by being present in the lab), not so much about what they can reveal about the underlying activities and the role of the digital media involved.

It is interesting that this part, which is supposed to focus on method, is so design-oriented, while only to some extent relying on classical methods for studying media interactions.

In the fifth and last part of the book, "Perspectives for Media Education in the Age of Digital Content Creation", Wildermuth's chapter "Empowerment" highlights the context of digital literacy in less fortunate parts of the world. Building on long-term fieldwork in Brazil, he underlines the need for digital empowerment rather than inclusion, aptly underscored by the recent events in Arab countries in North Africa and the Middle East, one might add. Simple technologies usually carry more value than sophisticated ones, especially when crime rates are high and income low. It is a sobering view from the other side of the digital divide. In the last chapter, Buckingham asks: "Do We Really Need Media Education 2.0?", representing another sceptical position in relation to the concept of the self-sufficient digital native. Denouncing the view that informal learning is of core importance in a new paradigm for learning, he insists that much has not changed in terms of media education as a critical method. Buckingham does not say so directly, but the tension between rapid new media distribution across society and the comparatively slow development of teaching practices involving digital media is at the root of the challenge he directs our attention to. And it is a welcome call for patience, although it may seem impossible at times.

With a broad selection of texts such as these, it can be hard to find a consistent thread throughout 15 chapters, but the organisation and the internal references do an excellent job of picking up overlapping perspectives across the contributions. The production quality of the book has a few proofing slips, enough to give it the feel of a digitally produced artefact. Perhaps it is a pertinent reminder that scholarly communication also might need an upgrade.

Finally, and actually most interestingly, it strikes me that this area of "digital content creation" is being increasingly described in designerly terms, i.e., by people blending traditional media studies with the growing body of design research. As a person who strongly believes in the value of both perspectives, to be frank, I find it only natural, and I see this book as a 
Book Review: Digital Content Creation - Perceptions, Practices \& Perspectives

solid sign that fruitful alliances harnessing the insights of both analytical and creative/productive communities should form the basis of coming research efforts on digital media in education, and in society at large, relying on theory-driven empirical research.

Martin Brynskov

Assistant Professor

Center for Digital Urban Living

Information and Media Studies

Aarhus University, Denmark

brynskov@imv.au.dk 